\title{
Attaching mass to the upper arm can increase throw distance in a modified javelin throw
}

\author{
NiCHOLAS LinTHORNE ${ }^{1 *}$, MARTIN HEYs ${ }^{1}$, TOMAS REYNOLDS ${ }^{1}$, NiLS ECKARDT $^{2}$ \\ ${ }^{1}$ Brunel University London, London, United Kingdom. \\ ${ }^{2}$ Carl von Ossietzky University of Oldenburg, Oldenburg, Germany.
}

\begin{abstract}
Purpose: The effectiveness of the whip-like coordination in throwing might be influenced by the inertial properties of the athlete's arm. This preliminary study investigated the acute effect of attaching mass to the upper arm on the distance achieved in a modified javelin throw. The aim was to identify the optimum upper arm mass that maximizes throw distance. Methods: Three well-trained adult male athletes performed maximum-effort throws with an 800 -g javelin training ball. A wide range of masses $(0-1.5 \mathrm{~kg})$ were attached to the upper arm and a 2D video analysis was used to obtain measures of the projection variables for each attached mass. Results: All three athletes showed an effect of attached arm mass on throw distance, and with the optimum mass the athlete's throw distance was increased by $2.2 \mathrm{~m}, 1.2 \mathrm{~m}$, and $0 \mathrm{~m}(7 \%, 4 \%$, and $0 \%)$, respectively. The optimum mass was specific to the athlete $(0.6 \mathrm{~kg}, 0.2 \mathrm{~kg}$, and $0 \mathrm{~kg})$ and changes in throw distance were mostly due to changes in release velocity rather than changes in release angle or release height. The experimental results were broadly similar to those obtained using a simple 2D mathematical model of throwing. Conclusions: These results indicate that some javelin throwers might see an increase in throwing performance when a mass is attached to their upper arm. However, the relationship between upper arm mass and throwing performance should be investigated further with studies on more athletes, projectiles of different mass, and other throwing events.
\end{abstract}

Key words: biomechanics, motion analysis, sport performance, throwing

\section{Introduction}

Several sports involve throwing a projectile at very high velocity. In sports like baseball and handball, velocity is used to get the projectile past an opponent, whereas in sports like the javelin throw, velocity is used to produce a long flight distance. The ability to launch a projectile with a very high velocity is mainly determined by the athlete's capacity to generate high muscular force and produce an effective pattern of coordination. As a consequence, most athletes in throwing sports spend considerable time training to improve their muscular strength and their throwing technique. An athlete's body dimensions are also important in throwing. An athlete with longer arm segments has a greater path length over which to acceler- ate the projectile and so tends to produce a faster throw. However, body segment lengths are fixed in an adult athlete and are not trainable. Other body segment parameters such as mass and moment of inertia can be substantially modified through muscle hypertrophy exercises (i.e., bodybuilding). In most humans the mass of the upper arm segment is about $70 \%$ greater than that of the forearm segment [6], and this mass difference is believed to enhance the whip-like coordination in a throwing movement and help produce a high projectile velocity [1], [13]. The present study was inspired by the prospect that performance in throwing sports might be improved through deliberate manipulation of the mass of the athlete's upper arm segment.

The present study investigated the acute effect of attaching mass to the upper arm on the distance

\footnotetext{
* Corresponding author: Nicholas Linthorne, Brunel University London, London, United Kingdom, Phone: +44 01895266479 , e-mail address: nick.linthorne@brunel.ac.uk

Received: March 12th, 2020

Accepted for publication: March 20th, 2020
} 
achieved in a modified javelin throw. The aim was to identify the optimum upper arm mass that maximizes throw distance. Our hypotheses were: 1) attaching mass to the athlete's upper arm affects throw distance; 2) changes in throw distance are due to changes in release velocity rather than changes in release angle or release height; and 3) the natural mass of the athlete's upper arm segment is not optimal and there is an optimum attached mass that maximizes the athlete's throw distance.

\section{Materials and methods}

This study used a single-subject experimental design in which the data from each participant was analyzed separately [2]. Three male decathletes volunteered to participate in the study (Table 1). The javelin throw is one of the ten component events in the decathlon and the participants were skilled in javelin throwing. Their javelin throw best performance was about $60 \%$ of the distance achieved by a specialist international javelin thrower [15]. The study adhered to the tenets of the Declaration of Helsinki and was conducted in accordance with procedures approved by our institutional ethics committee. We informed the participants of the procedures and inherent risks prior to their involvement in the study and we obtained their written informed consent.

Table 1. Characteristics of the participants

\begin{tabular}{|l|c|c|c|}
\hline \multicolumn{1}{|c|}{ Characteristic } & Participant 1 & Participant 2 & Participant 3 \\
\hline Age [years] & 20 & 22 & 21 \\
\hline Height [m] & 1.81 & 1.83 & 1.86 \\
\hline Body mass [kg] & 75 & 83 & 76 \\
\hline $\begin{array}{l}\text { Javelin throw } \\
\text { best performance [m] }\end{array}$ & 49.31 & 45.91 & 47.97 \\
\hline
\end{tabular}

The throws by the participants were performed in an indoor athletics facility in a single test session. The participants performed a modified javelin throw where they launched an 800-g javelin training ball (Nocken Ball, Gill Athletics, Champaign, IL, USA) from a twostep run-up to a throwing line. The short run-up reduced confounding factors associated with inter-trial variations in the participant's run-up velocity, and the training ball removed confounding factors associated with the aerodynamics of a javelin [3]. The participants had previous experience with throwing a training ball from a short run-up as part of their training program. The participants wore their own athletic training clothes (tight-fitting shorts and shirt/singlet) and javelin throwing shoes. Before commencing the test session, the participant performed his usual warmup routine for a javelin training session, which consisted of stretching, jogging, short sprints, and submaximal-effort throws with a training ball.

In the test throws, the participant's body position at the start of the run-up was regulated by instructing him to start with his throwing arm extended straight and back behind his body, with the angle of the arm slightly below the horizontal. The participant was also instructed to use his usual short run-up javelin throwing action with the goal of achieving maximum distance. It was assumed that the participant would throw at maximum effort with a self-selected release angle and release height.

Pilot testing indicated that the maximum tolerable attached mass was about $0.8-1.5 \mathrm{~kg}$, and a participant could perform up to 15-25 maximum-effort throws in a single test session. For the main study, steel or gel masses were attached to the upper arm segment of the throwing arm at about the midpoint of the segment using flexible bandaging tape. The throw conditions for participant 1 were (a) no attached mass, (b) $0.250 \mathrm{~kg}$, (c) $0.500 \mathrm{~kg}$, (d) $0.750 \mathrm{~kg}$, and (e) $1.500 \mathrm{~kg}$. The throw conditions for participants 2 and 3 were (a) no attached mass, (b) $0.225 \mathrm{~kg}$, (c) $0.450 \mathrm{~kg}$, and (d) $0.675 \mathrm{~kg}$. The participant performed 3-5 throws for maximum distance for each throw condition, and the order of the throw conditions was randomized to preclude any effect resulting from the order. Before each throw condition the participant was allowed several practice throws to become accustomed to the attached mass. The throw distances were measured to the nearest $0.1 \mathrm{~m}$ relative to the throwing line using a fiberglass tape measure, and the participant was given a rest interval of about 2-4 minutes between throws to minimize the effects of fatigue on throwing performance.

\section{Video analysis}

A 2D video analysis was conducted to determine the effect of attached arm mass on the participant's release variables. A JVC GR-DVL 9600 video camera (Victor Company of Japan, Yokahama, Japan) operating at $100 \mathrm{~Hz}$ was used to record the movement of the participant and ball during the throws. The video camera was placed at right angles to the throw direction and about $22 \mathrm{~m}$ away from the plane of the throw. The field of view was zoomed to allow the participant and ball to be in the field of view throughout the runup and throw and for at least 10 frames after release. The movement space of the video camera was calibrated with three vertical poles that were placed along the plane of the run-up and initial flight of the ball. 
An Ariel Performance Analysis System (Aerial Dynamics, Trabuco Canyon, CA, USA) was used to manually digitize the motion of the ball and the participant in the video images. The center of the ball and 18 body landmarks that defined a 17 -segment model of the participant were digitized in each image, starting from about 10 frames before touchdown of the delivery stride through to when the ball left the field of view of the camera. The segmental data used were those proposed by Dempster [6] for male adults. The two-dimensional coordinates of the ball, the body landmarks, and the participant's center of mass were calculated from the digitized data using the twodimensional direct linear transform (2D-DLT) algorithm. Coordinate data were smoothed using a secondorder Butterworth digital filter with a cut-off frequency of $6 \mathrm{~Hz}$ for the horizontal direction and $7 \mathrm{~Hz}$ for the vertical direction, and the velocity of the ball, the body landmarks, and the participant's center of mass were calculated by numerical differentiation of the coordinate data.

The instant of release was defined as the first frame in which the ball broke contact with the participant's hand. The release height was the vertical distance of the center of the ball relative to the ground at the instant of release, and the release distance was the horizontal distance of the center of the ball relative to the throwing line at the instant of release. The effective throw distance was calculated by adding the release distance to the measured throw distance.

The mean release velocity of the ball was obtained by fitting curves to ball displacement data from the first ten images immediately after the instant of release. The horizontal component of the ball velocity was the first derivative of a linear regression line fitted to unfiltered ball displacement data, and the vertical component of the ball velocity was the first derivative of a quadratic regression line (with the second derivative set equal to $-9.81 \mathrm{~m} / \mathrm{s}^{2}$ ) fitted to unfiltered ball displacement data [20]. The release velocity and release angle of the ball were calculated using the Pythagorean equation and the trigonometric tangent function. In this study all throws landed within $3^{\circ}$ of a line projected along the line of the run-up and so a $2 \mathrm{D}$ video analysis was expected to produce sufficiently accurate measures of release velocity and release angle.

\section{Data analysis}

To determine the participant's optimum attached upper arm mass, the participant's effective throw distance $(R)$ was plotted against mass $(m)$ and curves were fitted to the data. We fitted a straight line, a sec- ond-order polynomial, and a symmetrical hyperbola. A straight line can be written as $R=d m+R_{0}$, where $d$ is the gradient and $R_{0}$ is the range at zero mass. A second-order polynomial can be written in the form of an inverted u-shape;

$$
R=R_{\mathrm{opt}}-c\left(m-m_{\mathrm{opt}}\right)^{2},
$$

where $m_{\mathrm{opt}}$ is the optimum mass, $R_{\text {opt }}$ is the throw distance at the optimum mass, and $c$ is a measure of the curvature [14]. A symmetrical north-south opening hyperbola can be written as

$$
\frac{\left(R-R_{0}\right)^{2}}{b^{2}}-\frac{\left(m-m_{0}\right)^{2}}{\mathrm{a}^{2}}=1
$$

where $m_{0}$ and $R_{0}$ are the coordinates of the centre of the hyperbola and $b / a$ is a constant that determines the slope of the asymptotes. We set $b=1$ and so the vertex of the hyperbola has coordinates $m=m_{0}=m_{\mathrm{opt}}$ and $R=R_{0}+1=R_{\text {opt }}$. The most appropriate curve (straight line, second-order polynomial, or symmetrical hyperbola) was decided by examining the distribution of the residuals and with calculations of the corrected Akaike's Information Criterion [17]. The peak in the most appropriate curve was taken as the participant's optimum mass.

We also determined the participant's optimum mass from the release velocity data. A javelin training ball is a moderately aerodynamic projectile [5], so at the release velocities expected in this study $(15-25 \mathrm{~m} / \mathrm{s})$ the throw distance was expected to increase almost linearly with increasing release velocity [25]. Therefore, the form of the relationship between release velocity and mass was expected to be the same as that between throw distance and mass. As before, curves were fitted to the release velocity versus mass data and the peak in the most appropriate curve was taken as the participant's optimum mass.

We tested for an effect of attached upper arm mass on the participant's release angle and release height. The release angle and release height were plotted against mass and a straight line and a second-order polynomial were fitted to the data. We also tested for an effect of mass on the participant's run-up technique. The variables investigated were the participant's run-up velocity, length of delivery stride, and body position at the instant of touchdown of the rear leg into the delivery phase. The participant's run-up velocity was taken as the change in the horizontal position of the center of mass over the duration of the flight phase of the last stride before the delivery phase [8], and the length of the delivery stride was taken as the horizontal distance between the ankle joints at the 
instant of touchdown of the front leg. The participant's body position at touchdown of the rear leg was investigated using the height of the shoulder (acromion process) of the throwing arm and the angle of the elbow (in the plane of the throw).

In this study, the uncertainties in the measured values of release velocity and release angle arose mainly from the uncertainties in the fit to the coordinate data for the flight of the ball. The uncertainties ( $95 \%$ confidence interval) were about $0.3 \mathrm{~m} / \mathrm{s}$ for release velocity and $0.8^{\circ}$ for release angle. A substantial source of uncertainty in the release height, release distance, and technique variables was expected to arise from the sampling frequency of the video camera, and this uncertainty was taken as one half of the difference between the value at the instant of interest and the value at one frame before the instant of interest [8]. The average uncertainties were: release height $-0.07 \mathrm{~m}$, release distance $-0.06 \mathrm{~m}$, length of the delivery stride $-0.01 \mathrm{~m}$, height of the shoulder at touchdown $-0.01 \mathrm{~m}$, and elbow angle at touchdown $-1^{\circ}$. The $95 \%$ confidence interval in the variables arising from re-digitizing a trial five times were: release velocity $-0.1 \mathrm{~m} / \mathrm{s}$, release angle $-0.2^{\circ}$, release height $0.01 \mathrm{~m}$, release distance $-0.01 \mathrm{~m}$, run-up velocity $0.1 \mathrm{~m} / \mathrm{s}$, length of the delivery stride $-0.01 \mathrm{~m}$, height of the shoulder at touchdown $-0.01 \mathrm{~m}$, and elbow angle at touchdown $-2^{\circ}$. The uncertainties listed above are less than the inter-trial variations in the release variables and technique variables, and so a $2 \mathrm{D}$ video analysis with a sampling rate of $100 \mathrm{~Hz}$ was sufficient for the purposes of the study.

\section{Mathematical model of throwing}

We examined the effect of upper arm mass on release velocity using a simple two-segment rigid-body model of throwing. The throwing model was obtained by modifying the model presented by Daish [4]. In the throwing model, the free upper extremity consisted of two long thin rods ("upper arm" and "forearm with hand"), and the projectile was a point mass at the distal end of the forearm segment (Fig. 1). The athlete exerts a large constant torque about the shoulder joint $\left(T_{s}\right)$ and a much smaller torque about the elbow joint $\left(T_{e}\right)$. The linear velocity of the projectile at any instant is obtained by solving the equations of motion for the shoulder and elbow joints;

$$
\begin{gathered}
A \frac{d^{2} \theta}{d t^{2}}+B \frac{d^{2} \phi}{d t^{2}} \cos (\phi-\theta) \\
-B\left(\frac{d \phi}{d t}\right)^{2} \sin (\phi-\theta)+T_{s}-T_{e}=0
\end{gathered}
$$

$$
\begin{gathered}
B \frac{d^{2} \theta}{d t^{2}} \cos (\phi-\theta)+B\left(\frac{d \theta}{d t}\right)^{2} \sin (\phi-\theta) \\
+C \frac{d^{2} \phi}{d t^{2}}+T_{e}=0,
\end{gathered}
$$

where $\theta$ is the angle of the shoulder joint, $\varphi$ is the angle of the elbow joint, and $t$ is time. Here, $A, B$, and $C$ are given by

$$
\begin{gathered}
A=I_{1}+m_{2} L_{1}^{2}+m_{p} L_{1}^{2} ; \quad B=m_{2} h_{2} L_{1}+m_{p} L_{2} L_{1} ; \\
C=I_{2}+m_{2} h_{2}^{2}+m_{p} L_{2}^{2},
\end{gathered}
$$

where $m_{p}$ is the mass of the projectile, $m$ is the mass of the segment, $L$ is the length of the segment, $I$ is the relevant moment of inertia of the segment, $h$ is the distance of the centre of mass of the segment from its proximal end, and the subscripts 1 and 2 refer to the upper arm and the forearm. In this model, the centre of mass of the segment is located midway along the length of the segment (i.e., $h=L / 2$ ). Therefore, the moment of inertia of the upper arm segment for rotation about its proximal end is given by $I_{1}=m_{1} L_{1}^{2} / 3$, and the moment of inertia of the forearm segment for rotation about its centre of mass is given by $I_{2}=$ $m_{2} L_{2}{ }^{2} / 12$.

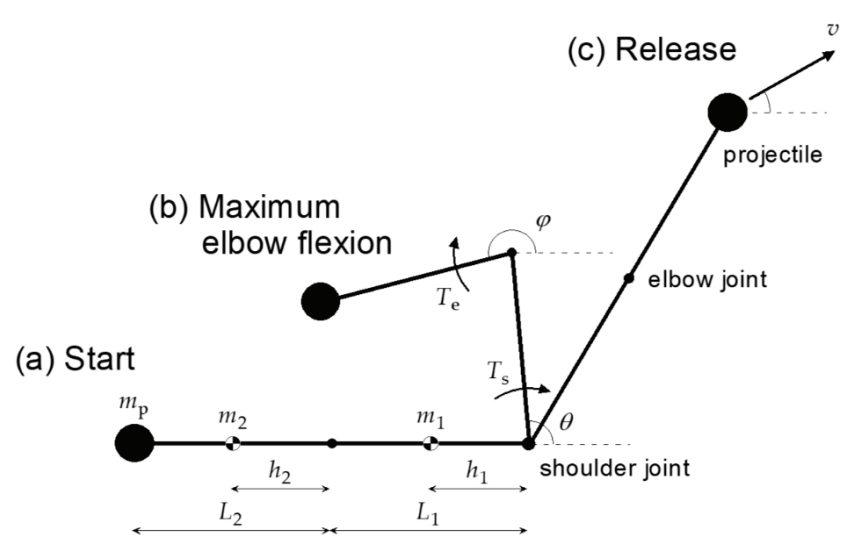

Fig. 1. Schematic diagram of a two-segment model of throwing a projectile for distance.

The arm is shown at the start of the throw (a), at the instant of maximum elbow flexion (b), and at the instant of release (c).

An explanation of the variables is given in the text

Equations (3) and (4) were solved to obtain curves for the angular position and angular velocity of the shoulder and elbow joints as a function of time. The linear velocity of the projectile $(v)$ at any instant was calculated using $v=r \omega$, where $r$ is the instantaneous distance of the projectile from the shoulder and $\omega$ is the instantaneous angular velocity of the projectile 
about the shoulder. Equations (3) and (4) are non-linear and so were computed using numerical methods that were implemented in a technical computing software package (Mathematica; Wolfram Research, Champaign, IL, USA). A "standard" athlete and projectile was selected with the following values: $m_{1}=2.0 \mathrm{~kg}$, $m_{2}=1.5 \mathrm{~kg}, m_{p}=0.8 \mathrm{~kg}, L_{1}=0.30 \mathrm{~m}, L_{2}=0.30 \mathrm{~m}$, $T_{s}=80 \mathrm{~N} \cdot \mathrm{m}$, and $T_{e}=10 \mathrm{~N} \cdot \mathrm{m}$. The mathematical model produced a broadly realistic simulation of throwing a javelin training ball. Initially, the throwing arm was held straight, horizontal, and motionless directly behind the athlete (Fig. 1). The shoulder torque caused the upper arm segment to rotate forwards; the elbow joint flexed and then extended again to the straightened $\left(0^{\circ}\right)$ position. The elbow joint rotated almost freely, but with this model a small torque at the elbow joint $\left(T_{e}\right)$ was necessary to produce a throw that had a realistic maximum elbow flexion angle (about $110^{\circ}$ ). In this study, the mass of the upper arm $\left(m_{1}\right)$ was systematically changed from the standard value, and the release velocity for each throw was noted. Simulations were performed for a projectile mass of $m_{p}=800$, $700,600,500$, and $400 \mathrm{~g}$, which correspond to the javelins used in competition by men, women, juniors, youths, and masters athletes.

\section{Results}

All three participants showed an effect of attached upper arm mass on throw distance (Fig. 2). For participants 1 and 2, the optimum upper arm mass that produced the greatest throw distance was greater than the athlete's natural mass, but for participant 3, the optimum attached mass was zero (Table 2; Supplementary Material). The effect of attached arm mass on release velocity was not as clear, but participants 1 and 2 again had an optimum mass that produced the greatest release velocity. For participants 1 and 2, a secondorder polynomial and a symmetrical hyperbola were equally the most appropriate fitted curve, and the optimum mass obtained from the release velocity data was similar to that obtained from the throw distance data.

The attached upper arm mass had little effect on the participant's release angle and release height (Supplementary Material). These results indicate that the observed changes in throw distance were mostly due to systematic changes in release velocity and were not due to systematic changes in release angle or release height. Attached arm mass also had little effect on the participant's run-up technique (Supplementary Material). The run-up velocity, length of delivery stride, and the shoul- der height and elbow angle at the instant of touchdown of the rear leg were about the same in all the throws. These results indicate that the observed changes in throw distance were not due to systematic changes in technique prior to the delivery phase of the throw.
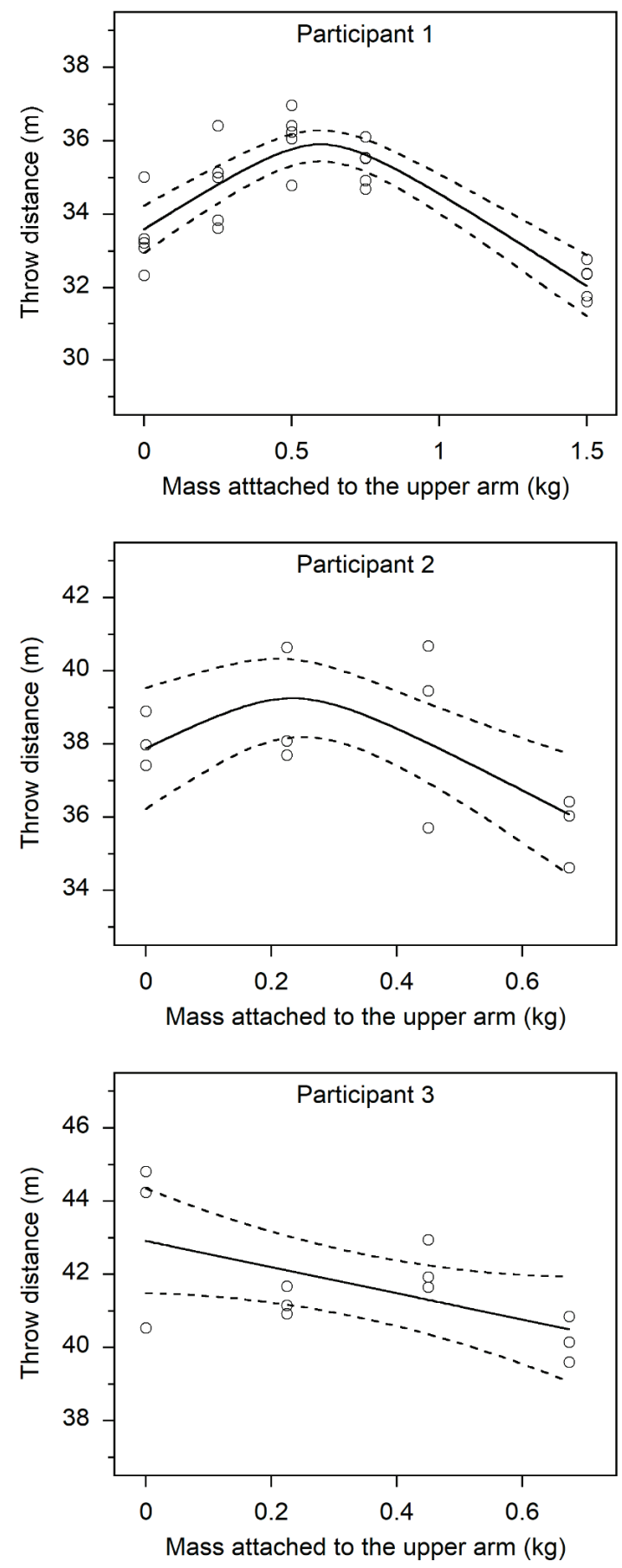

Fig. 2. These plots show the effect of added arm mass on throw distance for the three participants. The optimum added mass was $0.6,0.2$, and $0 \mathrm{~kg}$, and the throw distance was increased by $2.2 \mathrm{~m}, 1.2 \mathrm{~m}$, and $0 \mathrm{~m}$ (for participants 1,2 , and 3 , respectively). The solid line is a regression fit

(hyperbola for participants 1 and 2; linear for participant 3) and the dashed lines show the $95 \%$ confidence bands of the regression curve 
Table 2. The optimum mass attached to the upper arm and the corresponding throw distance $( \pm 95 \% \mathrm{CI})$, calculated from the throw distance data with a hyperbolic fit for participants 1 and 2 and with a linear fit for participant 3

\begin{tabular}{|l|c|c|c|}
\hline & Participant 1 & Participant 2 & Participant 3 \\
\hline $\begin{array}{l}\text { Optimum attached } \\
\text { upper arm mass [kg] }\end{array}$ & $0.60 \pm 0.08$ & $0.23 \pm 0.17$ & 0 \\
\hline $\begin{array}{l}\text { Throw distance } \\
\text { at optimum mass [m] }\end{array}$ & $35.9 \pm 0.6$ & $39.3 \pm 1.8$ & $42.9 \pm 1.4$ \\
\hline $\begin{array}{l}\text { Increase in throw } \\
\text { distance [m] }\end{array}$ & $2.5 \pm 1.3$ & $1.2 \pm 2.3$ & 0 \\
\hline
\end{tabular}

The throw simulations with the mathematical model revealed an effect of upper arm mass on release velocity, and there was an optimum upper arm mass that produced the greatest release velocity (Table 3; Supplementary Material). For an $800 \mathrm{~g}$ projectile, the optimum additional upper arm mass was $2.0 \mathrm{~kg}$, but the increase in release velocity was relatively small $(0.15 \mathrm{~m} / \mathrm{s} ; 0.9 \%)$. The optimum arm mass increased with increasing projectile mass.

Table 3. Increasing the mass of the projectile increased the optimum additional upper arm mass

\begin{tabular}{|c|c|c|}
\hline $\begin{array}{c}\text { Projectile mass } \\
{[\mathrm{g}]}\end{array}$ & $\begin{array}{c}\text { Optimum additional } \\
\text { upper arm mass }[\mathrm{kg}]\end{array}$ & $\begin{array}{c}\text { Release velocity } \\
{[\mathrm{m} / \mathrm{s}]}\end{array}$ \\
\hline 400 & -2.17 & 21.5 \\
\hline 500 & -0.57 & 20.3 \\
\hline 600 & 0.55 & 19.4 \\
\hline 700 & 1.38 & 18.6 \\
\hline 800 & 2.03 & 17.9 \\
\hline
\end{tabular}

\section{Discussion}

Our data mostly support the initial hypotheses. Attaching masses to the upper arm segment affected the throw distance for all three participants, and the changes in throw distance were mostly due to changes in release velocity and were not due to changes in release angle, release height, or run-up technique. For two of the three participants, the natural mass of their upper arm segment was not optimal and the optimum attached mass that maximized the athlete's throw distance was identified. However, one participant achieved the best throw distance with no attached mass. The results from the mathematical model were in broad agreement with the experimental data.

When throwing with no attached mass, the throw distances were about $70-90 \%$ of the participant's best competition javelin throw performance. This was probably because of the short run-up and the absence of flight enhancing aerodynamics in the training ball. When throwing with no attached mass, the average release angles $\left(30-35^{\circ}\right)$ and release heights $(2.1-2.4 \mathrm{~m})$ of the three participants were similar to those observed in competition javelin throw performances. The release angles for the participants in the present study were expected to be close to their optimum release angles. The optimum release angle in a javelin throw is less than $45^{\circ}$ because the release velocity an athlete can generate decreases as release angle is increased [14], [21].

Southard [23] reported results from an experimental study of the effect of attaching masses to the upper arm on throwing performance. He investigated the movement patterns of young adult men when throwing an underweight baseball $(0.114 \mathrm{~kg})$. The study was looking at whether attaching mass to the arm segments produces a qualitative change in the throwing pattern. Like the present study, Southard found that attaching mass to the upper arm could increase release velocity in some participants. He found that with an additional $1.4 \mathrm{~kg}$ strapped on the upper arm, the peak velocity of the hand during the throw was increased by about $15 \%$ in the most highly skilled participants. However, Southard's study used untrained participants performing a generic throwing action, whereas our study examined skilled athletes in a throwing sport.

Skillful throwing can be seen as an effective use of the "kinetic chain". A person with a more skillful sequencing of actions tends to throw faster because more of the work performed by the joint torques is converted into the kinetic energy of the projectile. Many studies have shown that highly skilled throwers use a proximal-distal sequence, where the peak values in joint torque and joint angular velocity occur later in the joints that are further along the kinetic chain towards the projectile. Expert baseball pitching and javelin throwing is powered by rapid sequential activation of many muscles, starting in the legs and progressing through the hips, torso, shoulder, elbow, and wrist [7], [24]. The torques generated at each joint accelerate the segmental masses, creating rapid angular movements that accumulate a high kinetic energy in the projectile at its release. This whip-like coordination is due to the dynamic coupling of the joints, whereby a torque at one joint induces angular acceleration at all joints in the system [9].

\section{Limitations}

The present study used a single-subject design with three participants. There have been misconceptions about the advantages and limitations of the single-subject approach [2]. Some have questioned the statistical power of this type of study and whether the low number of participants limits the generalizability of the findings. 
A single-subject analysis involves a detailed testing of a single participant using many trials and can produce strong evidence in support or against a particular hypothesis, as applied to that participant. In the present study, the power to detect an effect was mainly determined by the number of trials by the participant and by the range in the independent variable (i.e., the range of mass attached to the upper arm). The generalizability of the findings was addressed through replication (i.e., testing the hypothesis on additional participants). For a study in which there are three participants who have been randomly chosen from a population, the probability that all three participants produce the same result is relatively small (less than 5\%) [2]. The participants in the present study were drawn from a homogeneous population (healthy young adult male decathletes) in which the relevant inter-individual differences were expected to be relatively small. However, we observed substantial inter-individual differences in the form of the relationship between attached arm mass and throw distance (i.e., a hyperbolic relationship for participants 1 and 2, and a linear relationship for participant 3). Therefore, the findings from the present study cannot be generalized to other decathletes; a similar study with more participants is needed to reveal the full range of possible responses to attached upper arm mass in decathletes. A similar study with heptathletes and specialist javelin throwers (both male and female) is needed to generalize the findings to the broader population of javelin throw athletes.

The present study used a $2 \mathrm{D}$ video analysis with a sampling rate of $100 \mathrm{~Hz}$. This choice produced measures of the ball release variables that were sufficiently accurate for the purposes of the study. However, when throwing a projectile, the arm segments move very rapidly and are not always in the plane of the run-up. Therefore, the present study could not provide accurate measures of the changes in the participant's throwing technique arising from the attached arm mass. We recommend further studies be conducted using 3D high-speed motion analysis to obtain joint segment kinematics and joint torques. This might reveal the mechanisms behind the observed changes in release velocity and throw distance when mass is attached to the upper arm. However, such a study might be difficult as the interactions of the force, timing, and direction of motion during the throw are expected to complicate the mapping of the kinetic causes of motion to the velocity of the projectile at release.

Another limitation of the present study is that we tested only one throwing sport (javelin throw) and only one projectile mass $(800 \mathrm{~g})$. Different throwing sports use projectiles with substantially different mass (e.g., baseball - $148 \mathrm{~g}$, cricket - $156 \mathrm{~g}$, softball - $180 \mathrm{~g}$, American football $-415 \mathrm{~g}$, water polo $-425 \mathrm{~g}$, handball $-450 \mathrm{~g}$, javelin -600 and $800 \mathrm{~g}$ ). The results from the throwing model suggest that the optimum arm mass might depend strongly on the mass of the projectile. For some sports, increasing the mass of the athlete's upper arm might reduce the release velocity rather than increase it.

The mathematical model of throwing used in this study has substantial limitations. Although some of the results from the throwing model were in broad agreement with the experimental data, the qualitative predictions from the model might not be reliable. The model has several substantial simplifications, and these might influence the behavior of the model in response to added arm mass. The throwing model is two-dimensional and so it does not consider humeral longitudinal rotation [22]. The model also does not consider the contributions of the run-up and trunk motions to the generation of release velocity [24], and does not consider the contribution of the hand segment, which influences the timing of release and the release angle in high-speed ball throwing [10]. We suspect that a complex three-dimensional throwing model is needed to accurately assess the effects of upper arm mass on throwing performance.

\section{Implications}

The present study investigated the effect of attaching mass to the upper arm on throwing performance in a modified javelin throw. Other throwing activities that could benefit from an increase in release velocity include baseball pitching, shooting at goal in team handball and water polo, and passing by the quarterback in American football. However, for some throwing sports attaching a weight to the athlete's upper arm might not be accepted by the sport governing body. In 2006, the International Association of Athletic Federations (IAAF), now World Athletics, introduced a rule that prohibits attaching weights to the athlete's body in any of the throwing events (shot put, discus throw, hammer throw, and javelin throw) [11]. Also, in team handball, the players are not permitted to wear objects that could be "dangerous to the players" [12], and this rule might be interpreted to include arm weights. However, in American football, a quarterback can wear a hard object as long as it is covered in foam [19], and in Major League Baseball, there is no explicit ban on the pitcher wearing arm weights [16].

The present study investigated the effect of attaching small masses to the upper arm segment. An alternative method of increasing the mass of the upper arm is through muscle hypertrophy training (i.e., bodybuilding). Hypertrophy training is a permitted training technique 
for all sports and so hypertrophy training aimed at increasing the mass of a thrower's upper arm is unlikely to be banned. Silicone implants or site enhancement oil could also be used to increase the mass of the upper arm. However, we suspect that these methods have a high risk of being prohibited by sports governing bodies.

The throw distance for one participant in the present study was improved by $2.5 \mathrm{~m}(7 \%)$ with the optimum attached mass. In the javelin throw, a 7\% improvement in performance would turn an athlete who just qualified for the final at the Olympic Games into a medal winner [15]. However, specialist javelin throwers might have more massive upper arms than the participants in the present study. Therefore, specialist javelin throwers could already be close to their optimum upper arm mass and so might have less potential for improvement in throw distance through increasing their upper arm mass. Indeed, some athletes might have to decrease the mass of their upper arm to reach their optimum mass.

Throwing is not the only sports movement that uses a whip-like coordination. A whip-like coordination is also used in kicking movements, and the mass difference between the thigh and shank segments is believed to enhance the kicking action [25]. We suggest that it might be possible to increase ball velocity in kicking by attaching masses to the thigh segment of the kicking leg. A penalty kick in soccer and a kick at goal in American football, rugby union, and rugby league are some sports actions that might benefit.

\section{Conclusions}

Adding mass to the upper arm might have a meaningful effect on javelin throw performance. The present study was a preliminary investigation and the relationship between arm mass and throwing performance needs to be investigated with further studies on more athletes, on projectiles of different mass, and on other throwing events.

\section{References}

[1] Alexander R.McN., The Human Machine, Natural History Museum, 1992.

[2] BAtes B.T., Single-subject methodology: An alternative approach, Med. Sci. Sports Exerc., 1996, 28 (5), 631-638, DOI: 10.1097/00005768-199605000-00016.

[3] Best R.J., Bartlett R.M., Sawyer R.A., Optimal javelin release, J. Appl. Biomech., 1995, 11 (4), 371-394, DOI: 10.1123/jab.11.4.371.

[4] DAish C.B., The Physics of Ball Games, English Academic Press, 1972.
[5] De Mestre N., The Mathematics of Projectiles in Sport, Cambridge University Press, 1990.

[6] Dempster W.T., Space Requirements of the Seated Operator, WADC-TR-55-159, Wright-Patterson Air Force Base, Dayton, OH, 1955.

[7] Fleisig G.S., Barrentine S.W., Zheng N., Escamilla R.F., ANDREWS J.R., Kinematic and kinetic comparison of baseball pitching among various levels of development, J. Biomech., 1999, 32 (12), 1371-1375, DOI: 10.1016/ S0021-9290(99)00127-X.

[8] HAY J.G., NOHARA H., Techniques used by elite long jumpers in preparation for takeoff, J. Biomech., 1990, 23 (3), 229-239, DOI: 10.1016/0021-9290(90)90014-T.

[9] Hirashima M., Yamane K., NAKAmura, Y., OHTSUKi T., Kinetic chain of overarm throwing in terms of joint rotations revealed by induced acceleration analysis, J. Biomech., 2008, 41 (13), 2874-2883, DOI: 10.1016/j.jbiomech.2008.06.014.

[10] HoRe J., WATTS S., TweEd D., Errors in the control of joint rotations associated with inaccuracies in overarm throws, J. Neurophysiol., 1996, 75 (3), 1013-1025, DOI: 10.1152/ jn.1996.75.3.1013.

[11] IAAF, IAAF Competition Rules 2006-2007, International Association of Athletic Federations, 2006.

[12] IHF, Rules of the Game, International Handball Federation, 2016.

[13] Kreighbaum E., Barthels M., Biomechanics: A Qualitative Approach for Studying Human Movement, 4th ed., Allyn and Bacon, 1996.

[14] LinTHORne N.P., PATEl D.S., Optimum projection angle for attaining maximum distance in a soccer punt kick, J. Sports Sci. Med., 2011, 10 (1), 203-214.

[15] Matthews P. (Ed.), Athletics 2017: The International Track and Field Annual, SportsBooks, 2017.

[16] MLB, Official Baseball Rules 2019, Major League Baseball, 2019.

[17] Motulsky H., Christopoulos A., Fitting Models to Biological Data Using Linear and Nonlinear Regression, Oxford University Press, 2004.

[18] MuraKami M., Tanabe S., IshiKawa M., Isolehto J., KomI P.V., ITO A., Kinematic characteristics of the javelin throw in elite and domestic level throwers, New Studies Athletics, 2005, 20 (Suppl.), 67-79.

[19] NFL, Official Playing Rules of the National Football League, National Football League, 2018.

[20] NunOme H., Ikegami Y,, KozaKai R., Apriantono T., SANO S., Segmental dynamics of soccer instep kicking with the preferred and non-preferred leg, J. Sports Sci., 2006, 24 (5), 529-541, DOI: 10.1080/02640410500298024.

[21] Red W.E., Zogaib A.J., Javelin dynamics including body interaction, J. Appl. Mech., 1977, 44 (3), 496-498, DOI: 10.1115/1.3424108.

[22] RoAch N.T., Lieberman D.E., Gill T.J. IV, PALMER W.E., GILL T.J. III, The effect of humeral torsion on rotational range of motion in the shoulder and throwing performance, J. Anat., 2012, 220 (3), 293-301, DOI: 10.1111/ j.1469-7580.2011.01464.x.

[23] Southard D., Mass and velocity: Control parameters for throwing patterns, Res. Quart. Exerc. Sport, 1998, 69 (4), 355-367, DOI: 10.1080/02701367.1998.10607710.

[24] Tidow G., Model technique analysis sheets - Part X: The javelin throw, New Studies Athletics, 1996, 11 (1), 45-62.

[25] Wesson J., The Science of Soccer, Institute of Physics Publishing, 2002. 


\section{Supplementary material}

\section{Experimental data}

\section{Results}

All three participants showed an effect of attached upper arm mass on throw distance (Fig. S1). For all three participants, there was an optimum mass that produced the greatest throw distance (Tables S1, S2, and S3). The effect of attached upper arm mass on release velocity was not as clear, but participants 1 and 2 had an optimum mass that produced the greatest release velocity (Fig. S1). For participants 1 and 2, the most appropriate fitted curves were a secondorder polynomial and a hyperbola, and for participant 3, the most appropriate curve was a straight line. The optimum masses obtained from the release velocity data were similar to those obtained from the throw distance data (Tables S1, S2, and S3).

The observed changes in throw distance were mostly due to systematic changes in release velocity and were not due to systematic changes in release angle or release height (Fig. S1; Table S4). For all three participants, a straight line was the best fit to the release height data. For participants 1 and 3, the gradient of the line was not substantially different from zero. For participants 2 and 3, a straight line was the best fit to the release angle data and the gradient of the line was not substantially different from zero. For participant 1, the release angle at the largest mass $(1.50 \mathrm{~kg})$ was slightly greater than those for the other masses. Across the other masses, a straight line was the best fit to the release angle data and the gradient of the line was not substantially different from zero.

Attached upper arm mass had little effect on the participant's run-up technique (Fig. S2). For all three participants, a straight line was the best fit to the runup velocity data and the length of delivery stride data, and the gradients of the lines were not substantially different from zero. Likewise, the technique variables at the instant of touchdown of the rear leg did not show any systematic variation with mass. The elbow flexion angle and the height of the shoulder of the throwing arm were about the same in all the throws. These results indicate that the observed changes in throw distance were not due to systematic changes in technique prior to the delivery phase of the throw.

The release values and technique values for the throws with no attached upper arm mass were similar to values reported for skilled male javelin throwers (Table S5) [S1], [S2].

\section{References}

[S1] Best R.J., Bartlett R.M., SAwyer R.A., Optimal javelin release, J. Appl. Biomech., 1995, 11 (4), 371-394.

[S2] Murakami M., Tanabe S., Ishikawa M., Isolehto J., Kомі P.V., ITO A., Kinematic characteristics of the javelin throw in elite and domestic level throwers, New Studies Athletics, 2005, 20 (Suppl.), 67-79. 
Participant 1
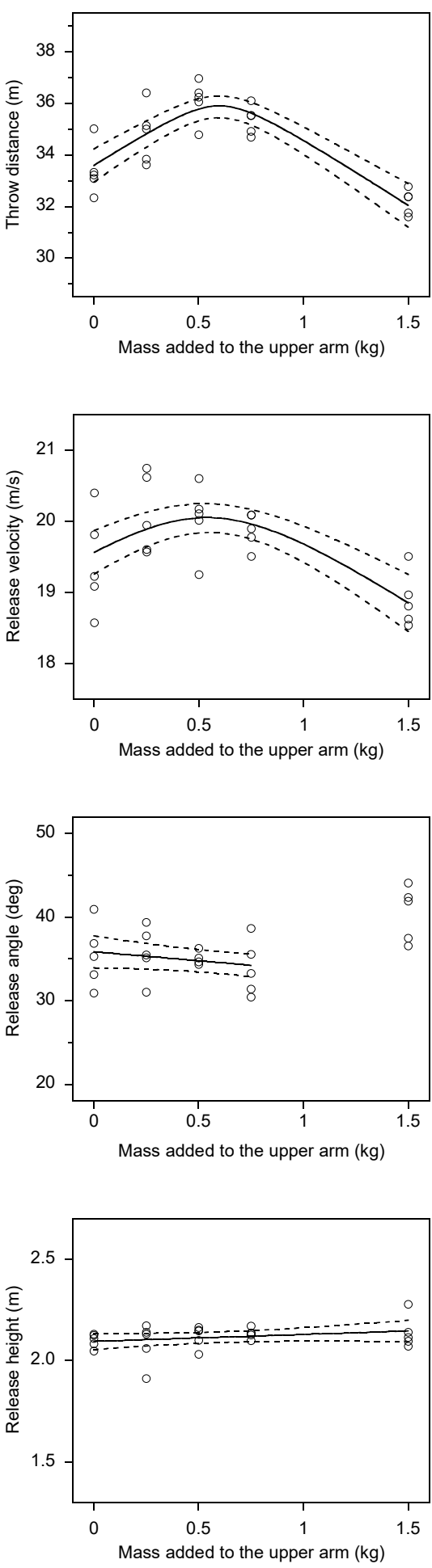

Participant 2
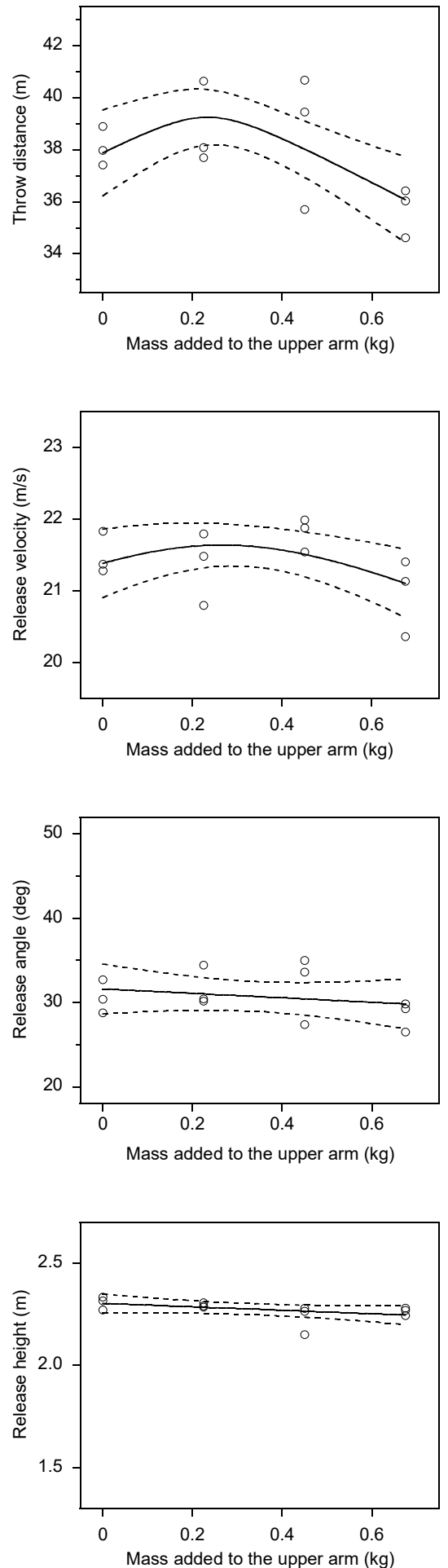

Participant 3
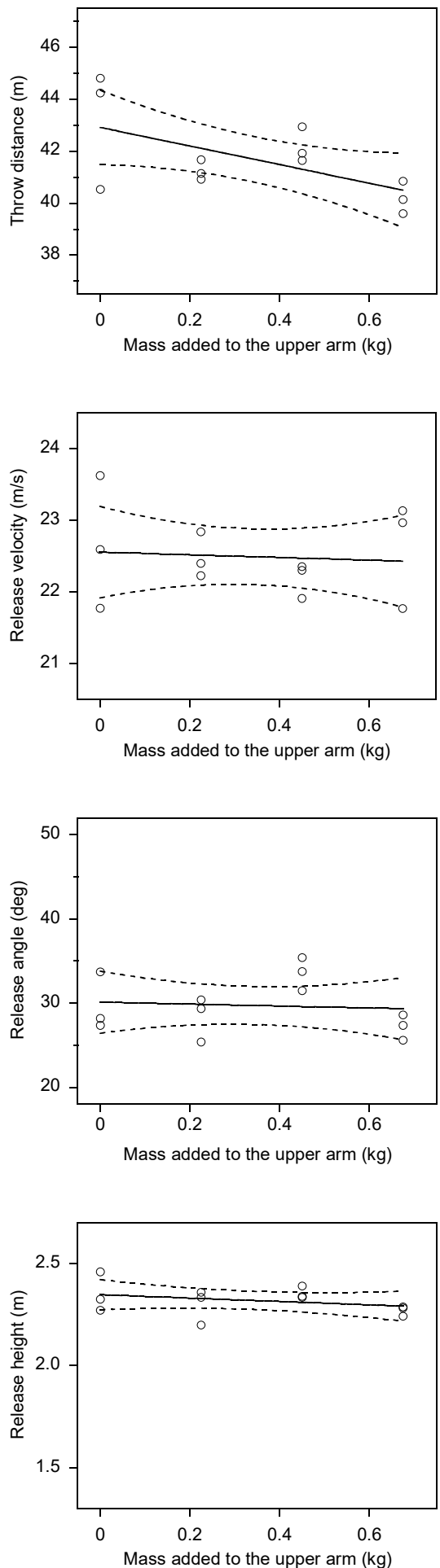

Fig. S1. These plots show the effect of the mass attached the upper arm on the throw distance for three participants.

Changes in throw distance were mostly due to changes in release velocity and were not due to changes in release angle or release height.

The solid line is a regression fit and the dashed lines show the $95 \%$ confidence bands 
Participant 1
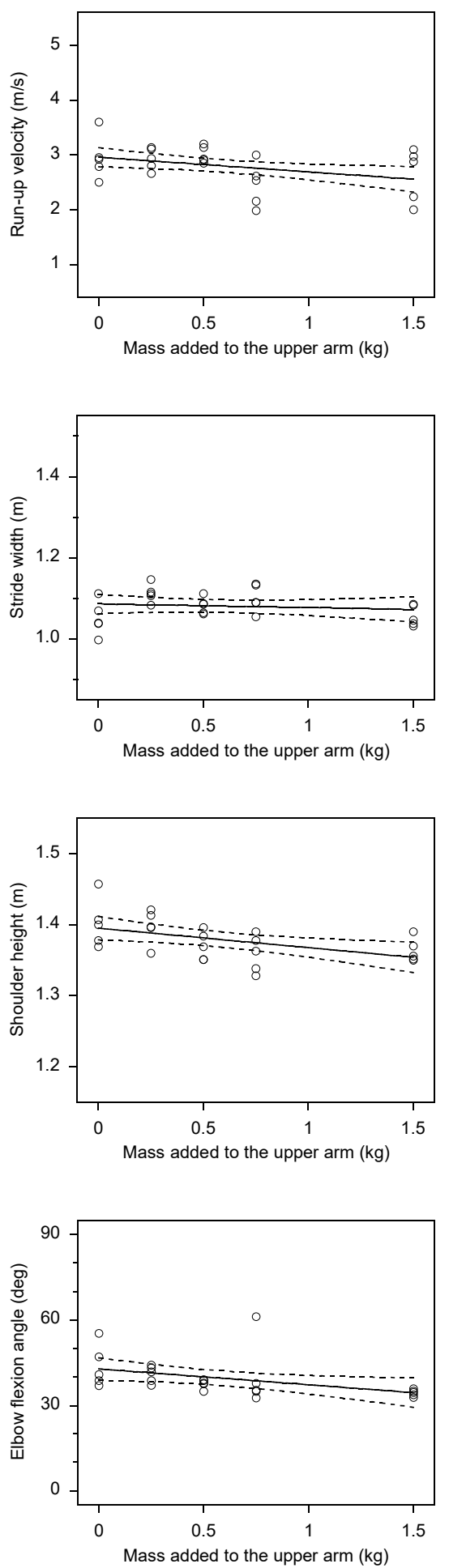

Participant 2
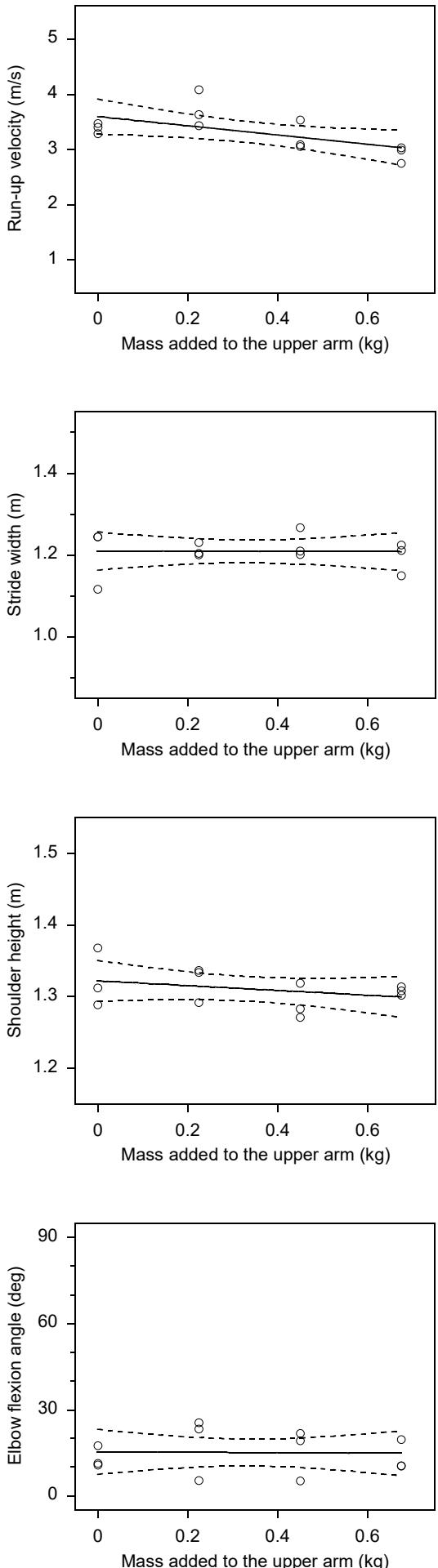

Participant 3
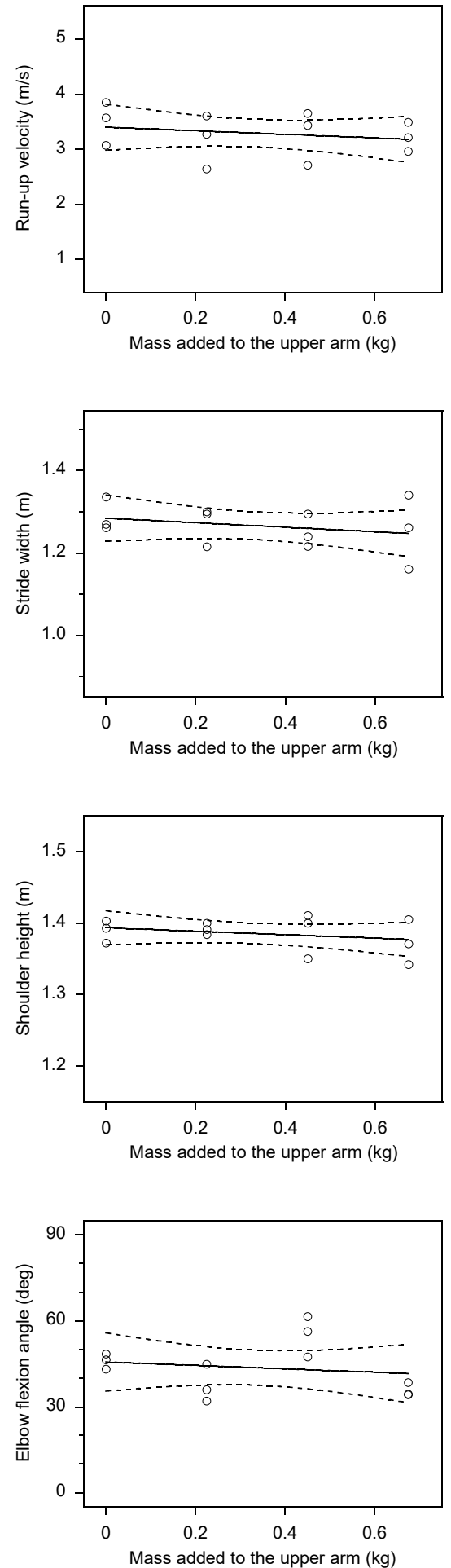

Fig. S2. The mass attached to the upper arm had little effect on the participant's run-up technique.

The solid line is a linear regression fit and the dashed lines show the $95 \%$ confidence bands of the regression curve 
Table S1. Participant 1: Optimum mass attached to the upper arm and the corresponding throw distance $( \pm 95 \% \mathrm{CI})$, calculated from a second-order polynomial fit Eq. (1) and a hyperbolic fit Eq. (2) to the throw distance data and release velocity data

\begin{tabular}{|l|c|c|}
\hline \multirow{2}{*}{\multicolumn{1}{|c|}{ Variable }} & \multicolumn{2}{c|}{ Participant 1 } \\
\cline { 2 - 3 } & polynomial & hyperbola \\
\hline Optimum mass [kg] from throw distance data & $0.66 \pm 0.07$ & $0.60 \pm 0.08$ \\
\hline Optimum mass [kg] from release velocity data & $0.59 \pm 0.18$ & $0.54 \pm 0.19$ \\
\hline Throw distance at optimum attached mass [m] & $35.8 \pm 0.5$ & $35.9 \pm 0.6$ \\
\hline Increase in throw distance [m] & $2.4 \pm 1.3$ & $2.5 \pm 1.3$ \\
\hline Increase in throw distance [\%] & $7.1 \pm 3.8$ & $7.5 \pm 4.0$ \\
\hline
\end{tabular}

Table S2. Participant 2: Optimum mass attached to the upper arm and the corresponding throw distance $( \pm 95 \% \mathrm{CI})$, calculated from a second-order polynomial fit Eq. (1) and a hyperbolic fit Eq. (2) to the throw distance data and release velocity data

\begin{tabular}{|l|c|c|}
\hline \multirow{2}{*}{ Variable } & \multicolumn{2}{c|}{ Participant 2 } \\
\cline { 2 - 3 } & polynomial & hyperbola \\
\hline Optimum mass [kg] from throw distance data & $0.25 \pm 0.15$ & $0.23 \pm 0.17$ \\
\hline Optimum mass [kg] from release velocity data & $0.26 \pm 0.21$ & $0.27 \pm 0.23$ \\
\hline Throw distance at optimum attached mass [m] & $39.1 \pm 1.5$ & $39.3 \pm 1.8$ \\
\hline Increase in throw distance [m] & $1.0 \pm 2.4$ & $1.2 \pm 2.3$ \\
\hline Increase in throw distance [\%] & $2.6 \pm 6.3$ & $3.0 \pm 6.1$ \\
\hline
\end{tabular}

Table S3. Participant 3: Optimum mass attached to the upper arm and the corresponding throw distance $( \pm 95 \% \mathrm{CI})$, calculated from a linear fit to the throw distance data and release velocity data

\begin{tabular}{|l|c|}
\hline \multicolumn{1}{|c|}{ Variable } & Participant 3 \\
\hline Optimum mass [kg] from throw distance data & $0^{*}$ \\
\hline Optimum mass [kg] from release velocity data & $-{ }^{* *}$ \\
\hline Throw distance at optimum attached mass [m] & $42.9 \pm 1.4$ \\
\hline Increase in throw distance [m] & 0 \\
\hline Increase in throw distance [\%] & 0 \\
\hline
\end{tabular}

* The optimum attached mass was $0 \mathrm{~kg}$ because the gradient was negative and the $95 \%$ confidence interval of the gradient did not include zero (Table S4).

** The optimum attached mass was not defined because the $95 \%$ confidence interval of the gradient included zero (Table S4).

Table S4. Gradient of a linear fit to the plots of release variables and technique variables as a function of attached arm mass $( \pm 95 \% \mathrm{CI})$

\begin{tabular}{|c|c|c|c|}
\hline Variable & Participant 1 & Participant 2 & Participant 3 \\
\hline Throw distance $[\mathrm{m}]$ & - & - & $-4 \pm 3$ \\
\hline Release velocity $[\mathrm{m} / \mathrm{s}]$ & - & - & $0 \pm 2$ \\
\hline Release angle $\left[{ }^{\circ}\right]$ & $-2 \pm 5$ & $-3 \pm 6$ & $-1 \pm 9$ \\
\hline Release height [m] & $0.03 \pm 0.05$ & $-0.08 \pm 0.11$ & $-0.08 \pm 0.18$ \\
\hline Run-up velocity $[\mathrm{m} / \mathrm{s}]$ & $-0.3 \pm 0.3$ & $-0.8 \pm 0.8$ & $-0.4 \pm 1.0$ \\
\hline Length of delivery stride [m] & $-0.01 \pm 0.03$ & $0.00 \pm 0.11$ & $-0.05 \pm 0.14$ \\
\hline Height of shoulder at touchdown [m] & $-0.03 \pm 0.02$ & $-0.03 \pm 0.07$ & $-0.02 \pm 0.06$ \\
\hline Elbow flexion angle at touchdown $\left[{ }^{\circ}\right]$ & $-6 \pm 5$ & $-1 \pm 19$ & $-6 \pm 24$ \\
\hline
\end{tabular}


Table S5. Mean value of the release variables and technique variables with no attached arm mass (mean $\pm 95 \% \mathrm{CI}$ )

\begin{tabular}{|l|c|c|c|}
\hline \multicolumn{1}{|c|}{ Variable } & Participant 1 & Participant 2 & Participant 3 \\
\hline Throw distance [m] & $33.4 \pm 1.2$ & $38.1 \pm 1.9$ & $43.2 \pm 5.8$ \\
\hline Release velocity [m/s] & $19.4 \pm 0.9$ & $21.5 \pm 0.7$ & $22.7 \pm 2.3$ \\
\hline Release angle $\left.{ }^{\circ}\right]$ & $35 \pm 5$ & $31 \pm 6$ & $30 \pm 9$ \\
\hline Release height [m] & $2.10 \pm 0.04$ & $2.31 \pm 0.08$ & $2.35 \pm 0.24$ \\
\hline Run-up velocity [m/s] & $3.0 \pm 0.5$ & $3.4 \pm 0.2$ & $3.5 \pm 1.0$ \\
\hline Length of delivery stride [m] [m & $1.05 \pm 0.05$ & $1.20 \pm 0.18$ & $1.29 \pm 0.10$ \\
\hline Height of shoulder at touchdown $[\mathrm{m}]$ & $1.40 \pm 0.04$ & $1.32 \pm 0.10$ & $1.39 \pm 0.04$ \\
\hline Elbow flexion angle at touchdown $\left[{ }^{\circ}\right]$ & $44 \pm 9$ & $13 \pm 9$ & $46 \pm 7$ \\
\hline
\end{tabular}

\section{Mathematical model of throwing}

\section{Results}
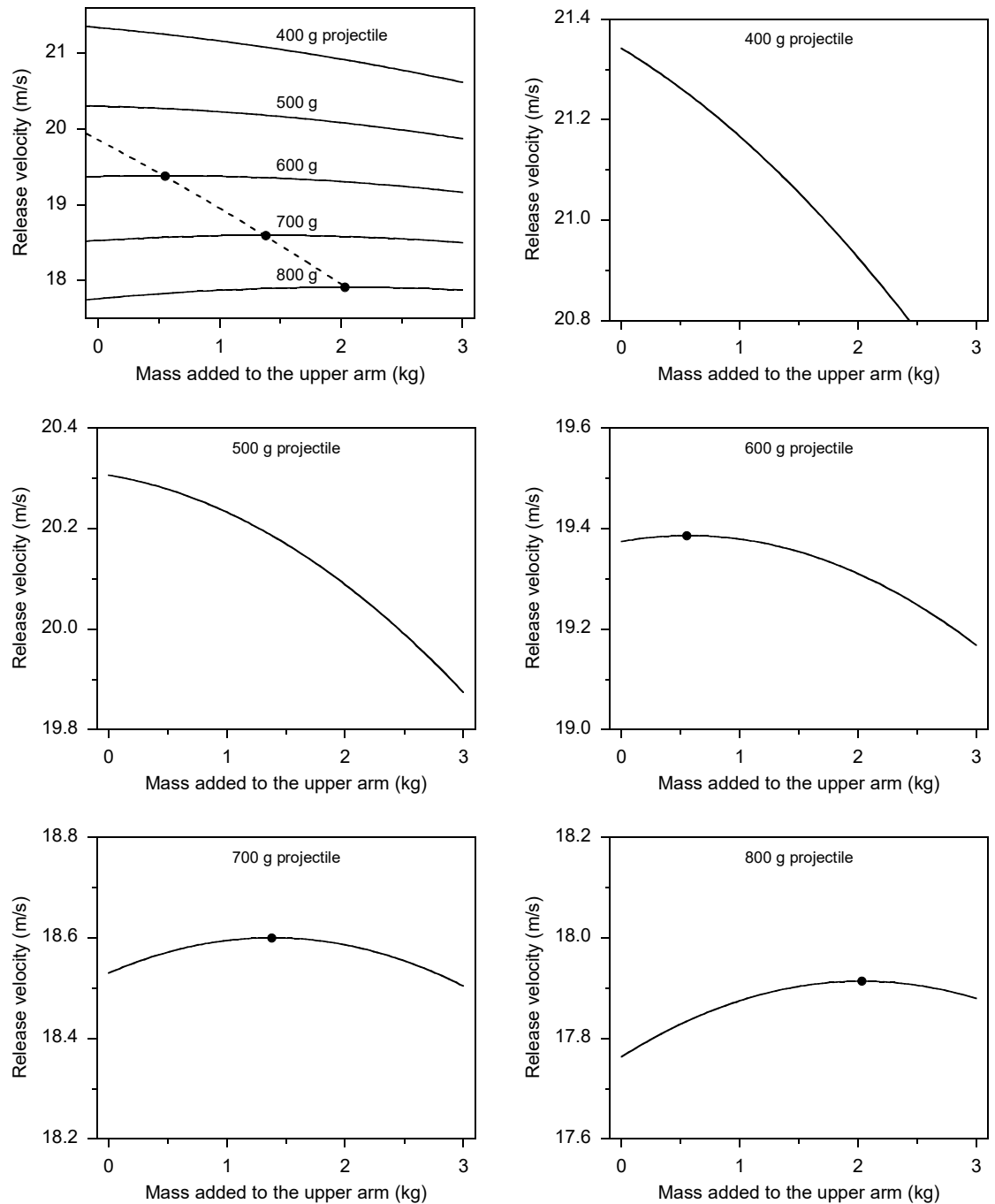

Fig. S3. Throw simulations with the 2D mathematical model revealed an optimum upper arm mass that maximizes release velocity. For an $800 \mathrm{~g}$ projectile, the optimum additional upper arm mass was $2.0 \mathrm{~kg}$, but the increase in release velocity was relatively small $(0.15 \mathrm{~m} / \mathrm{s} ; 0.9 \%)$.

The optimum additional mass (indicated by the "dot") increased with increasing projectile mass 